\title{
Managing Infrastructure Asset: Bayesian Networks for Inspection and Maintenance Decisions Reasoning and Planning
}

\author{
Haoyuan Zhang*, D. William R. Marsh \\ Risk and Information Management Research Group, School of Electronic Engineering and \\ Computer Science, Queen Mary University of London, London, E1 4NS, UK
}

\begin{abstract}
Models of maintenance problems must handle complex assumptions, allowing, for example, the condition of some assets to be rated directly using multiple states while in others the condition rating is inferred from that of the components from which they are assembled. The overall condition inferred, which informs the maintenance decisions, requires evidential reasoning under uncertainty. This paper uses Bayesian networks to address these challenges with real case studies. We apply the binary factorisation technique to allow inference of multi-state condition prediction, and further extend it to predict the condition of an asset with multiple components. These models are used to recommend inspection decisions such as which assets to inspect and when to inspect them. Models are also developed to evaluate the effectiveness of repair interventions and to use this to suggest repair actions. We show how to model multiple interventions within the asset life cycle considering both repair effectiveness and further deterioration. This modelling allows us to plan maintenance activities for an asset over its whole life cycle.
\end{abstract}

Keywords: Condition prediction, Multi-state system, System configuration, Bayesian networks, Maintenance modelling, Observational and intervention

\section{Decisions to Manage Infrastructure Asset}

As of 2017, the National Bridge Inventory (NBI) database in the United States showed that 54,560 bridges (out of a total of 615,002 ) were reported as structurally deficient (SD). The associated expenditure for maintenance is considerable - for

\footnotetext{
${ }^{*}$ Corresponding author.

Email address: haoyuan.zhang@qmul . ac.uk (Haoyuan Zhang)
} 
instance, the Organisation for Economic Co-operation and Development (OECD) estimated that from 2010 to 2030, the annual cost of road and rail maintenance would be approximately USD 220 billion and USD 50 billion respectively [1]. Furthermore, Heng et al. [2] estimated that with current practices, more than a third of infrastructure maintenance expenditure is wasted through poor decisionmaking. The need for an effective maintenance strategy for managing such decisions is therefore paramount.

With infrastructure asset, an effective maintenance strategy requires careful planning of both inspection and maintenance activities. Inspection aims to detect any damage and identify the underlying condition of the asset so that future inspection and maintenance can be scheduled. Alternatively, maintenance is performed to either repair any damaged that has occurred or to improve the overall condition to help prevent any future damage. These two activities ensure the infrastructure asset operates within an acceptable level of safety and reliability. An effective maintenance strategy is therefore necessary for increasing the expected life expectancy of structure and reduce its maintenance expenditure [3].

\subsection{Inspection Decisions}

Inspection activity can be classified into two types: visual inspection and detail inspection. Visual inspection looks for outward signs of damage, such as missing fasteners and cracks, which include techniques like fault detection and fault pattern recognition (see a review from Heng et al. [2]). While detail inspection seeks to determine the conditions of the underlying parts of the infrastructure, which is often more complicated to perform and generally more expensive. For example, for a bridge with hidden critical element that cannot be observed visually, we may need to use intrusive or non-intrusive examination methods to estimate its condition. We can perform a preliminary assessment by predicting its deterioration to support the decision on performing detail inspection.

The process of deterioration, such as a bridge deteriorates from a good condition to a structurally deficient condition, describes the decrease in asset condition over time. At the same time, historical inspection records provide information about the previous condition of an asset over time. We can use these records to infer relevant data to model the deterioration process of an asset. By doing so, we can predict the condition of an asset by estimating how soon it is likely to deteriorate into an unacceptable level in the future. This can support the decisions on inspection and answer questions like when to inspect the asset or which asset we should inspect. 


\subsection{Maintenance Decisions and Plans}

After we identified the damage or condition of an asset from inspection activity, we can decide its corresponding maintenance activity. Decisions on maintenance activity mainly involve with the selection of repair action and the schedule of maintenance. Repair actions can range from no action when the asset is in an acceptable state, to replacement when the asset on the edge of failure, with several intermediate actions to restore the asset to a better decay level [4]. We may want to suggest repair action given an objective, for example, minimum cost or an acceptable level of system reliability.

The choice of repair action is one of the keys to plan maintenance. Sometimes, from a life-cycle point of view, it is more cost-effective to wait for an asset to deteriorate further before taking an repair action instead of repairing it immediately. For example, we may have a longer life expectancy to repair an asset in a worse condition using a major repair action, than repair with minor repair action in a better state. Planning maintenance over a time horizon concerns with multiple intervention cycles, where each cycle considers the selection of repair action and the further deterioration after repair. As a result, the maintenance plan can support decisions like maintenance resource allocation. Or even, together with an optimisation technique, to provide an optimal selection of repair actions within its life cycle given an objective.

\subsection{Paper Outline}

This paper provides solutions to support the decisions addressed above. In Section 2, we introduce traditional reliability modelling approaches and the advantages of Bayesian Network (BN) models in supporting complex system modelling and decisions reasoning. Section 3 presents a variety of inspection and repair decision models using the Bayesian approach. These decision models are applied from Section 4 to 6 using real-world case studies. The paper ends with the conclusion in Section 7.

\section{Techniques for Reliability Analysis}

Fault tree analysis and event tree analysis are two commonly used modelling techniques when making asset reliability-related decisions. However, they often fail to model complex interaction between events and the structures of them increase exponentially with the increase in the number of states or components [5, 6], which limits their uses in maintenance decisions. Another popular approach is Markov-based stochastic modelling. A Markov process is a stochastic 
process where the future state only depends on the present state (Markov property). For example, Cesare et al. [7], Jiang et al. [8] applied Markov models to reason the deterioration of an asset with a sequence of state transition, and Shafahi and Hakhamaneshi [9] use it to model a maintenance management system. We can also extend it to a semi-Markov process which enables the modelling of an asset with multiple states where each transition between states follows a different distribution as shown in Droguett et al. [10]. But as summarised in Yianni et al. [11], this approach also suffers limitations in maintenance modelling, such as assuming a fixed discrete time interval and static transition probabilities.

Petri Net (PN) is capable of modelling discrete events with concurrencies and complex dependencies, and it has drawn more and more attention recently in reliability modelling [12, 13]. For example, Andrews [14] modelled the deterioration, inspection and maintenance processes of a track section with PNs. Also, we can extend PN to Coloured PN (CPN) to distinguish between tokens, which can greatly reduce the model space for complex structures. When modelling multiple components, we can share the same subnet to track multiple components using different coloured tokens [15]. Le and Andrews [16] used CPN to build a bridge management system with multiple components. Within each component, the PN models the non-constant deterioration and maintenance process, which are later combined as a complete bridge model. These models also support decisions such as prediction of lifetime condition of components or bridges, or implementation of a strategy like opportunistic maintenance [15, 16, 11]. To evaluate different maintenance strategies and to perform life-cycle cost analysis, Le [15] coupled PN with a genetic algorithm to optimise the selection of repair actions based on different repair effectiveness and costs. Monte Carlo (MC) simulation is often used for estimating the transition probabilities within PN. But with the increase in the number of components and structural hierarchy, the computational cost increases exponentially. This challenge can be addressed by parallelisation and accelerated by Graphics Processing Units, either parallelise the PNs with independent subnets like Chalkidis et al. [17], or parallelise the MC simulations like Yianni et al. [18].

Another alternative technique is BN. In a BN model, causal or influential relationships between variables are specified by a directed graph and a set of variables. Its joint probability distribution is calculated using the following equation:

$$
p(X)=\prod_{i=1}^{n} p\left(X_{i} \mid \operatorname{parents}\left(X_{i}\right)\right)
$$

Rafiq et al. [19] developed a discrete $\mathrm{BN}$ to infer the bridge condition from 
its components, but these conditions were directly given by experts. While Littlewood and Verrall [20] used continuous BN to learn component failure distributions from data. Reasoning decisions from a $\mathrm{BN}$ may require the use of both discrete variables (e.g. predicted state) and continuous variables (e.g. statistical distribution) in the same $\mathrm{BN}$ [13], this type of $\mathrm{BN}$ is called hybrid BN. Many inference algorithms were developed recently for hybrid BN. For example, an approximate inference algorithm was proposed in Marquez et al. [21] that combines dynamic discretisation with propagation algorithm on junction tree. It was applied to learn components failure with various distributions, and these distributions were used to diagnose the system performance with the help of discrete variables. This algorithm was implemented in the tool AgenaRisk [22], and in this paper, we use this tool for its flexibility and efficiency in BN modelling and inference.

Extended from Marquez et al. [21], a Bayesian-based condition prediction model that learns asset deterioration process from both data and knowledge was developed in Zhang and Marsh [23], and was recently validated and further extended to learn from similar asset groups in Zhang and Marsh [24]. Figure 1] presents a simplified model. The time each asset transits from one state to another state was modelled following a Weibull distribution. The transition distribution is inferred from past transition of assets in the same class (e.g. assets 1 to 4 for transition from Good to Fair). Each prior for shape and scale parameter follows a triangular distribution, which can be used to express knowledge about the deterioration of this type of asset. For example, if an engineer believes the deterioration rate of this asset increases over time, we can assign a shape value that is greater than 1 . 


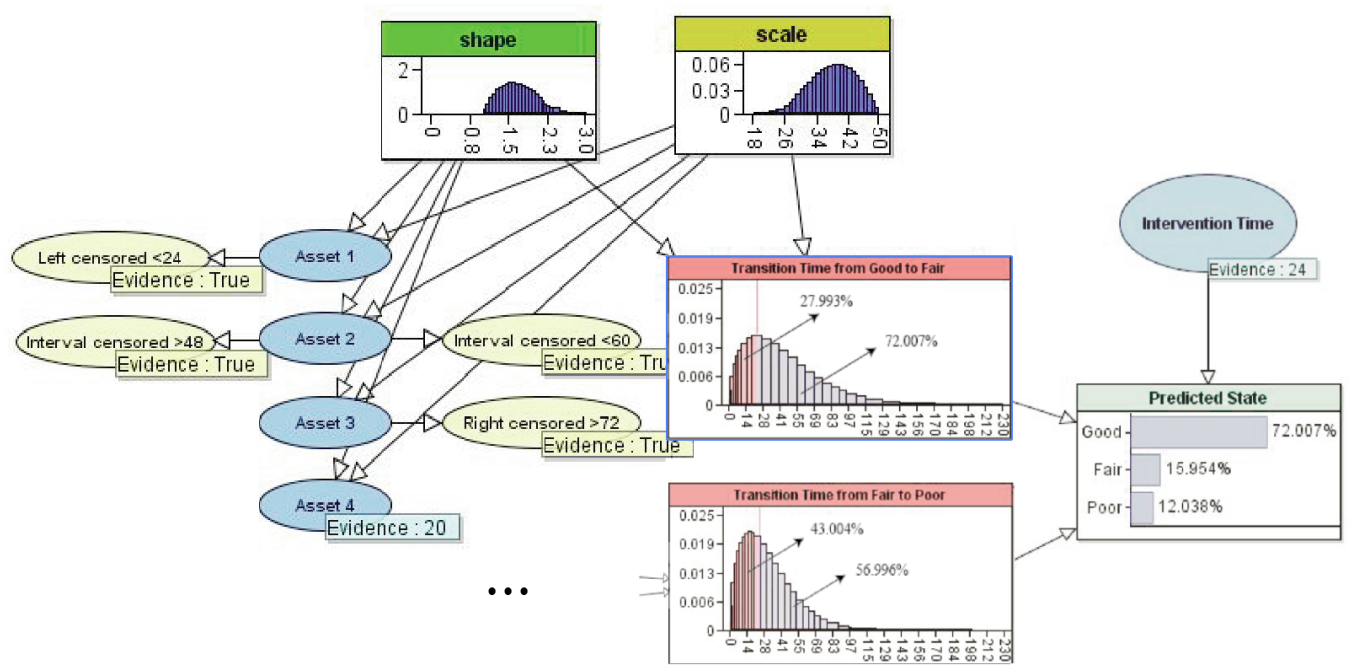

Figure 1: An asset condition prediction model.

We can use the learned transition distributions to infer asset state given an observation. As an example, an asset rated by three states is modelled using a categorical variable with two transitions from Good to Fair $\left(T_{\text {Good } \rightarrow \text { Fair }}\right)$ and from Fair to Poor $\left(T_{\text {Fair } \rightarrow \text { Poor }}\right)$ is shown in the same figure. Each transition is modelled by a separate parameter learning model. We assume deterioration progresses with the sequence of the rating system, that is, the transition from Good to Poor must go through the transition of Fair. Hence, denotes intervention time as $T$, in this example, the expression for the predicted state node becomes if $\left(T<T_{\text {Good } \rightarrow \text { Fair }}\right.$, "Good", if $\left(T<T_{\text {Fair } \rightarrow \text { Poor }}\right.$, "Fair", "Poor")). Assuming the starting state of this asset is a Good condition, the query from $T$ will first visit $T_{\text {Good } \rightarrow \text { Fair. }}$. In the probability density function of this node, only $27.993 \%$ of the area of this distribution is smaller than 24 , that means, $72.007 \%$ of this asset will still stay at Good state. For those transit to Fair, only $43.004 \%$ will further transit to the Poor state showed in $T_{\text {Fair } \rightarrow \text { Poor }}$, that is, $27.993 \% * 43.004 \%=12.038 \%$. This gives us the condition prediction distribution as shown in node predicted state.

Based on the condition prediction model discussed above, this paper develops a variety of Bayesian-based decisions models to manage infrastructure asset. We show not only we can model complex structures efficiently, but also reason a wide range of inspection and maintenance-related decisions. Further, we demonstrate the use of these models using real-world case studies. 


\section{Bayesian Networks for Asset Maintenance Modelling}

In this section, we extend the condition prediction model proposed in Zhang and Marsh [23, 24] to a range of BN models that can address various asset maintenance problems. Section 3.1 shows how to infer asset inspection conditions efficiently and with complex dependencies. Section 3.2 gives two models to support the process of making repair decisions. Section 3.3 shows how to plan maintenance activities with multiple intervention cycles. This section is further summarised in Section 3.4.

\subsection{Models for Inspection Decisions}

The aforementioned model can infer an component's condition distribution given an intervention interval. This subsection discusses how to perform inference more efficiently when we have a higher number of states and how to model assets that are assembled by multiple components.

\subsubsection{Binary Factorisation for Multi-State Component}

Figure 1 shows a simple example with only three states. In practice, we often have a much larger number of states, such as a component is graded by a 10-point scale in the NBI dataset (see Weseman [25]). With the increase in the number of state, the number of parent nodes for predicted state node increases correspondingly. In a BN, a node that has too many parents results in a very large Conditional Probability Table (CPT), which leads to computational complexity for its inference. Especially for continuous variables like the transition distribution, this complexity increases sharply since dynamic discretisation algorithm discretises each distribution into dozens of states [26]. If, say 30 states were discretised for each transition, for the NBI dataset, the predicted state would have 9 transition parents, leading to a request of $30^{9}$ entries for the CPT.

To reduce the inference complexity, here we adopt the binary factorisation modelling technique proposed in Neil et al. [27]. This technique converts the aggregation structure in the $\mathrm{BN}$ into a binary tree structure (for example, from Figure 2 (a) to (b)) that aims to ensure all nodes in the BN have no more than two continuous nodes as parents. In our model, the intervention time node and all transition nodes are continuous variables. Therefore, for the first transition, we build a temporal Boolean node (showed in dotted line) to query if the component transits to worse states given the intervention time. The following temporal Boolean nodes have the same structure, only with an additional link from the previous temporal node in the form of a Markov model. It will transit to worse states only if the previous state is transited to the current state and current transition is smaller than the 


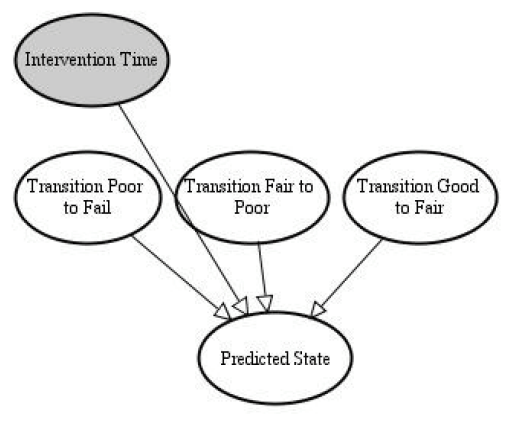

(a)

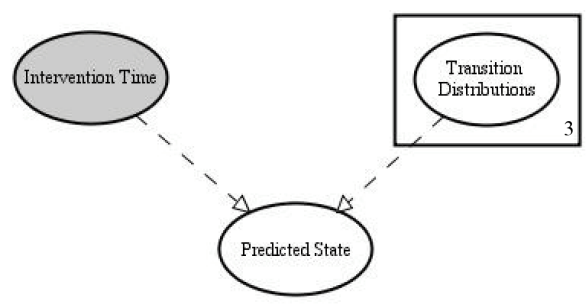

(c)

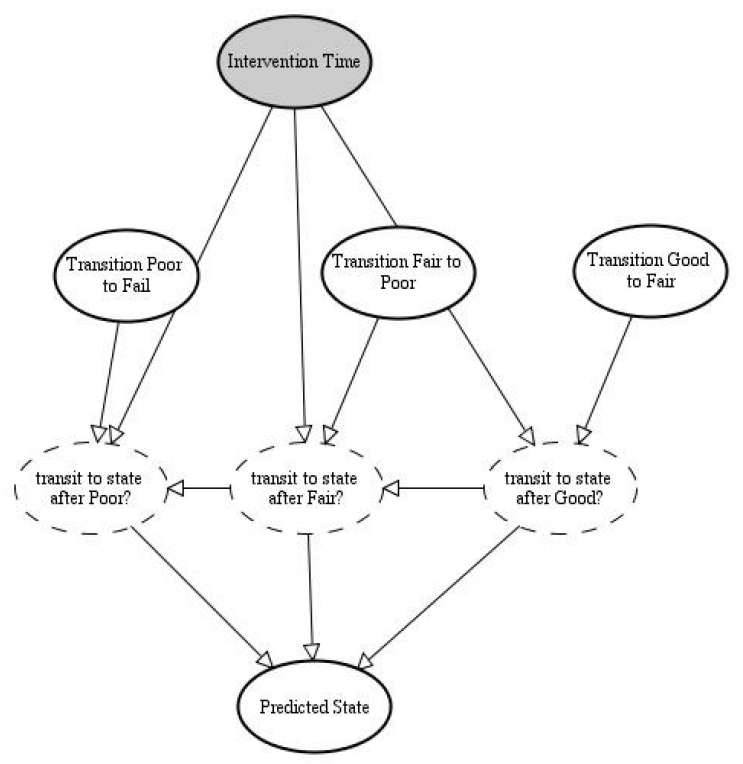

(b)

Figure 2: Condition prediction of a multi-state component: (a)model with aggregation; (b)binary factorised model; (c)plate model.

intervention time. For example, denotes node transit to state after Good as $t_{\text {Good }}$, the second temporal transition node is true when $\left(t_{\text {Good }}=\right.$ True $) \wedge\left(T>T_{\text {Fair } \rightarrow \text { Poor }}\right)$. At last, all the temporal nodes are linked to the predicted state node. This creates a much smaller CPT than the one with the aggregation structure: these temporal nodes are discrete variables with only two states for each. If the rating system has $n$ states, the binary factorised model reduces the CPT size of an aggregated structure from $30^{(n-1)}$ to $2^{(n-1)}$. In the example in Figure 2 , it reduces from $30^{3}$ to $2^{3}$, and for the NBI example, it can reduce the space from $30^{9}$ to $2^{9}$.

This modelling technique is employed in the rest of the paper. Figure 2 (c) gives an example of how to represent the model in (b) using a modified plate model to make the models more readable: a square indicates that there are multiple variables inside the square with the same structure. The index shows the number of variables with the same structure. The temporal nodes are omitted but represented by the dotted lines, indicating the model is binary factorised. 


\subsubsection{Assets with Multiple Components}

The condition of an asset with multiple components can be evaluated in various ways depending on the type of interaction between its components, such as in parallel, series, and their variants. Dynamic Fault Trees (DFTs) is one of the most notable frameworks in modelling these system configurations. Marquez et al. [26] represent the DFTs in the form of event-based BN structure to model parallel and series system. The transition distribution of an asset system is characterised by its components' transitions using an arithmetic function, such as maximum function for a parallel configuration and minimum function for a series configuration.

However sometimes, decision makers are more interested in evaluating the state of an asset from the state of its components, rather than directly from the transition distribution of its components. For example, in the US, one criterion in determining whether a bridge is structurally deficient is a condition rating of 4 or less for any of its structures, including deck, superstructure, substructure and culvert (if exists). We can consider each structure as a subsystem of the bridge, and they are assembled with a parallel configuration.

In a simple case where assets are rated by binary states (for example, working or fail), we can model them as traditional FT with a Boolean node in the BN. An AND gate can be used to model the relationship of a parallel system. It represents the event fails if all the components in the system fail. Similarly, an OR gate can be used to model the relationship of a series system. It represents the event fails when at least one of the components fails. The Boolean node is constructed with a comparative statement to express these logic gates and can be used to perform static FT analysis. For example, for a binary-state asset that is evaluated by the states of two components $\mathrm{C} 1$ and $\mathrm{C} 2$ in parallel, we can define: Asset $\sim$ if $(\mathrm{C} 1==$ "Fail" $\& \& C 2==$ "Fail", "Fail", "Working"). With BN tools like AgenaRisk [22], this expression for the Boolean operator can be automatically transformed to a corresponding CPT for inference in the BN. The joint probability distribution of the BN model that has a collection of independent cause variables Component $\underline{\mathrm{C}}$ consists of Component $C_{i}$, where $i=1,2, \ldots, n$ as the parents of the asset's state Y:

$$
p(\underline{C}, Y)=p(Y \mid \underline{C}) \prod_{i=1}^{n} p\left(C_{i}\right) .
$$

Therefore, together with the prior/posterior probability of component state (assigned directly from inspection data or learned from the parameter learning model explained earlier), we can get the state distribution of a binary-state asset with parallel or series configuration after inference. Thought we could extend the if- 
else statement in a Boolean node into a collection of logic expressions (nested if-else statements) in a categorical node to evaluate multi-state asset, the process for defining these logic expressions becomes tedious with the increase in the number of state.

Instead, for a multi-state asset that is evaluated by multi-state components, functions such as maximum or minimum can be used to describe the relationships between states of components and state of the asset. To do that, we can represent the states with numerical scales rather than simply using categorical states. Here, their states are modelled with a set of ordinal continuous intervals. The discrete variable (state of components and assets) is mapped onto a continuous scale that is bounded (from 0 to 1 ) and monotonically ordered. The reason for expressing them on an ordinal scale is that asset states are ordered. For example, in the NBI, State 8 represents a closer condition to State 9 than State 7 to State 9. Given the number of asset states, we can discretise the numerical scale accordingly. Hence, the binary-state system becomes one of the special cases with an interval of 0.5. Figure 3 presents a three-state scale example: the interval between each state is 0.333. Thus, Good state belongs to the interval of $[1,0.667)$ and Fair state belongs to the interval of $[0.667,0.333)$ and so forth.

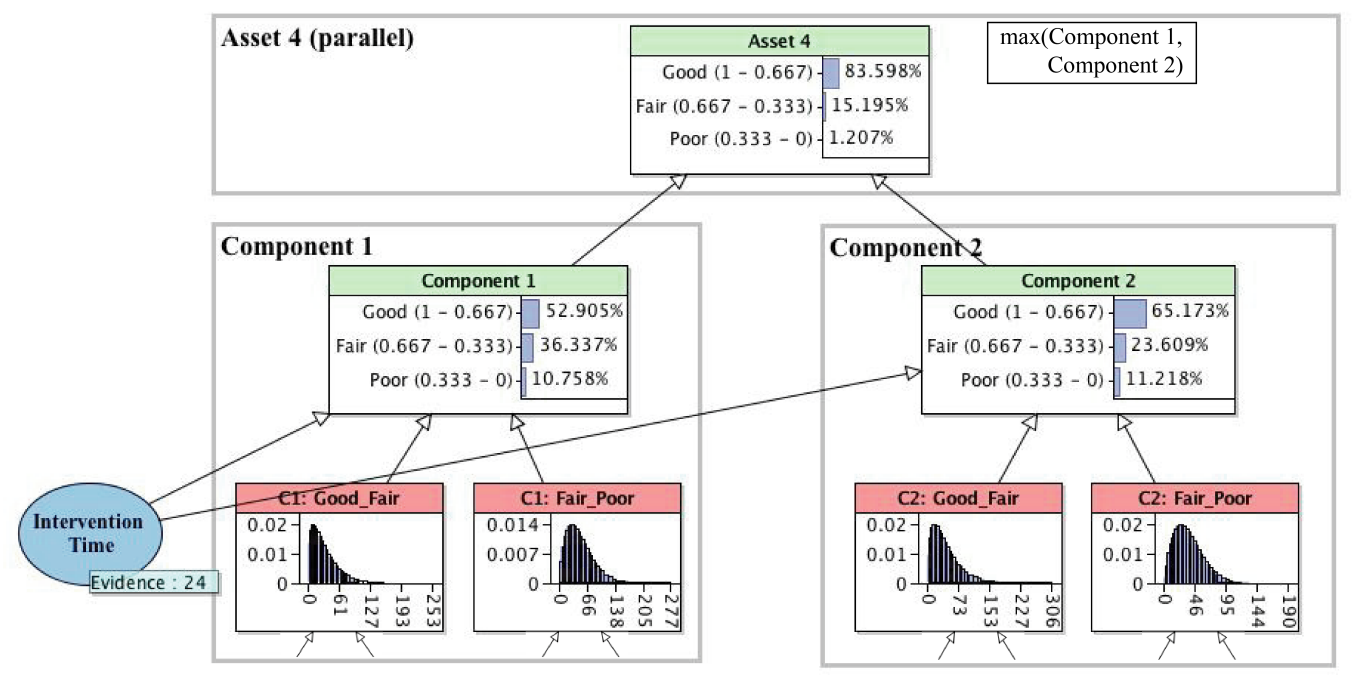

Figure 3: Multi-state asset system assembled by parallel components.

With these numerically scaled states, we now can use maximum and minimum functions. Since the states, for example, Good to Poor, are mapped to ordered numerical bounds from high to low, and their states are mutually exclusive, the 
probability of asset under maximum or minimum function becomes the maximum or minimum state scale of their components and then re-marginalised. Figure 3 presents an example of two components assembled in parallel. The CPT generated from the maximum function for asset 4 is given in Table 1 . The probability of the asset follows the sequence of logic: if either component is in Good state, the asset is in Good state, or if either component is in Fair state, the asset is in Fair state, otherwise, the asset is in Poor state.

Table 1: CPT for an asset with two components that are assembled in parallel.

\begin{tabular}{ccccccccccc}
\hline & Component 1 & \multicolumn{3}{c}{ Poor } & \multicolumn{3}{c}{ Fair } & \multicolumn{3}{c}{ Good } \\
\cline { 2 - 10 } & Component 2 & Poor & Fair & Good & Poor & Fair & Good & Poor & Fair & Good \\
\hline \hline \multirow{3}{*}{ Asset 1 } & Poor $(0-0.333)$ & 1.0 & 0.0 & 0.0 & 0.0 & 0.0 & 0.0 & 0.0 & 0.0 & 0.0 \\
& Fair $(0.333-0.667)$ & 0.0 & 1.0 & 0.0 & 1.0 & 1.0 & 0.0 & 0.0 & 0.0 & 0.0 \\
& Good $(0.667-1)$ & 0.0 & 0.0 & 1.0 & 0.0 & 0.0 & 1.0 & 1.0 & 1.0 & 1.0 \\
\hline
\end{tabular}

\subsection{Models for Repair Decisions}

When the state of an asset has been identified, we would like to decide what repair action to perform. This subsection develops two types of supports for making these repair decisions: one for suggesting maintenance actions based on historical repair usage, which is built upon a type of $\mathrm{BN}$ called an observational model; one for evaluation of repair effectiveness, which is built upon a type of BN called an intervention model. Both models are built with a common cause with confounder causal structure, which represents a situation that an event is causally affecting both the cause and the effect [28, 29].

\subsubsection{Observational Model: Historical Frequency of Repair Action}

Given an observed condition of an asset, maintainers may want to know under the same scenario, what action would other maintainers take. Figure 4 presents an example observational model for a three-state asset. Three repair actions are included for illustration purpose, this variable is the child node of the observed asset state since historically, the decision on repair action depends on the current state of the asset. These two nodes together become the parent nodes of the repaired state. In addition, we also include a constraint node to evaluate if the repaired state is still in Poor condition. 


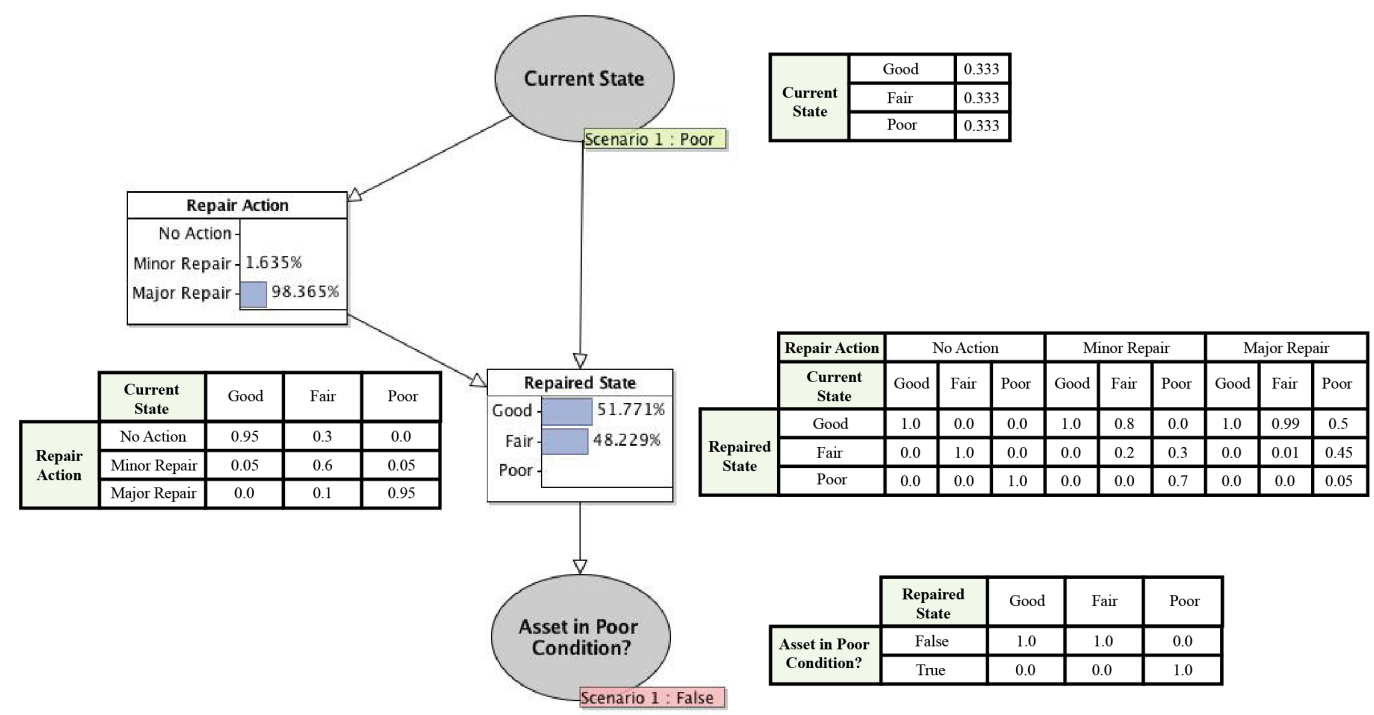

Figure 4: Observational model for repair action suggestion from historical frequency.

The CPT of current state node is uniformly distributed, but in most cases, this node is observed. In this example, it is observed with a Poor condition. The CPT of repair action depends on the observed state. Its entries are adopted from the historical frequency of repair action usage or expert judgement. For example, when the asset is inspected with a Fair condition, in the repair history, $30 \%$ of maintainers took no action, $60 \%$ used minor repair, and $10 \%$ applied major repair. Similar procedure is applied to specify the CPT of the repaired state about maintenance effectiveness. This example assumes the available actions are imperfect maintenance, and different actions have different effects in restoring asset state depending on its current state. For example, in history, 99\% of assets in Fair conditions repaired by major repair actions were restored to Good conditions while $1 \%$ didn't have any effect; $50 \%$ of them successfully restored asset in Poor condition to Good condition, and $45 \%$ to Fair condition but 5\% failed to improve its current state. The constraint node is an observable Boolean node with a logical expression if (Repaired State == "Poor", "True", "False"), and its equivalent CPT is shown in the figure. The False state is observed in this node to prevent the repaired state from being in a Poor condition.

By observing the state of the asset and the constraint to prevent asset from being in an unreliable condition, with this observational model we can infer the repair action frequency that was taken in history or from expert experience. In Figure 4, for an asset that is currently in Poor condition, in order to avoid its 
repaired condition still in the Poor condition, in history, $1.635 \%$ of maintainers took minor repair action and $98.365 \%$ repaired with major repair.

\subsubsection{Intervention Model: Effectiveness of Maintenance}

An intervention model can be used to answer questions such as what is the condition distribution of an asset if a specific repair action was taken. The distribution implies imperfect maintenance that not all actions can restore asset condition to a specific state, but a collection of states probabilistically.

Figure 5 presents an example of how to transform an observational model (Figure 4) into an intervention model for repair action evaluation. Followed the suggestions from Constantinou et al. [30], in the intervention model, the link from the cause should be removed. In the observational model, this link is used to explain the observation for repair intervention; while in the intervention model, we need to remove it in order to avoid inferring posterior probability for cause (current state node) when performing intervention (observed). Though technically this does not have an impact on the example in Figure 5 because both current state node and repair action node are observed. It is still necessary to remove this correlation because the current state could be predicted instead of being observed (see an example later given in maintenance planning). With this link, the model will wrongly reason backwards and have an impact on the current state. Furthermore, the constraint node becomes unobservable; it acts as an indication of the intervention impact.

Figure 5 shows an example of estimating the impact of an intervention. Most CPTs for the transformed intervention model remain the same as those in the observation model, except for the CPT of the repair action. The repair action node becomes independent from current state node and is observed. Hence, like the current state node, its CPT is uniformly distributed. For an asset in a Poor condition, performing a minor repair only has a $30 \%$ probability restoring the asset to Fair condition yet $70 \%$ probability staying in the Poor condition. Hence, in this case, we may suggest using major repair action instead.

In this subsection, we modelled a simplified relationship between repair actions and asset state. The model can be extended to describe more complex maintenance assumptions, such as some repair actions are only applicable to restore asset in a specific range of state, or repair actions depend on the states of multiple components. We can also include more constraints, such as costs and expected service time, to provide a reference for making maintenance decisions under a more practical scenario. These complex decisions are later discussed in Section 5 using a real example. Also, by considering the intervention model as a fragment 


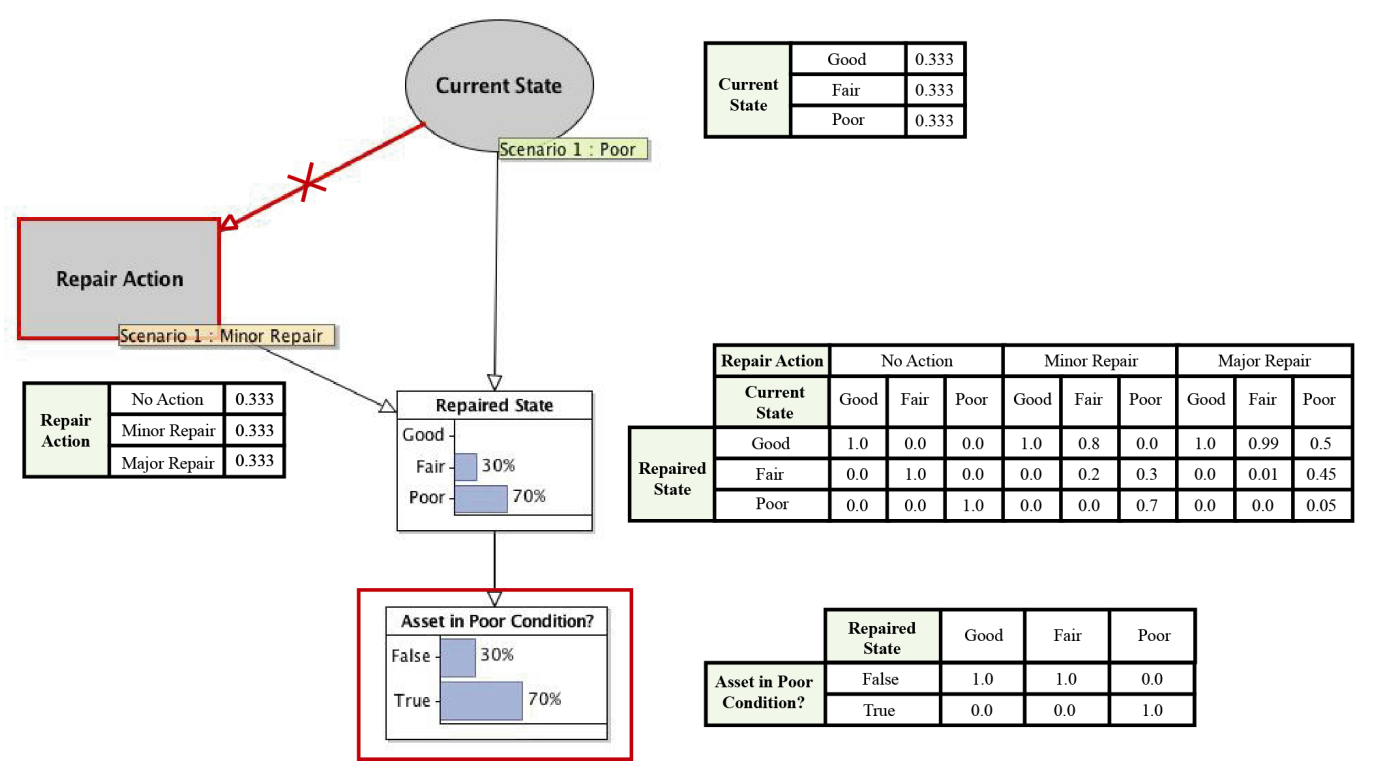

Figure 5: Converting an observational model to an intervention model for repair effectiveness.

model, we can form multiple fragments to model multiple maintenance decisions. Together with the condition prediction model from Section 3.1, we can plan the maintenance over a time period, and further, perform analysis such as life cycle analysis.

\subsection{Models for Maintenance Planning}

Intervention model from the last subsection gives us a tool to evaluate the repair effectiveness but does not explicate decisions should be taken to optimise the use of resources automatically. An adequate approach is needed to formalise the process of making these decisions, especially with the increase in complexity in decisions making of many problems, such as a situation where future decisions depend on past decisions [31]. In this subsection, the maintenance decision process is modelled as an object (fragment) that contains the intervention model together with the condition prediction model. By modelling multiple objects that are arranged sequentially, it allows us to model decision processes over a finite time period and perform analysis such as a life-cycle cost analysis.

For critical infrastructure, inspection and maintenance are often performed periodically. Therefore, to model a lifetime maintenance process, we can model multiple intervention processes that are either carried out with a fixed time interval of a dynamic time interval. Each intervention process is represented as an object 
that consists of the decision on repair action and its further deterioration till the next intervention. We can connect multiple intervention processes in the form of sequentially organised BN objects. In each object, the posterior of predicted state becomes the prior of the predicted state of its next object (next intervention). The repair action is a decision node, it is determined by the state of the asset's further deterioration (for example, the most probable state or a state is above a certain percentage) from the last intervention. The repair cost is cumulatively calculated depending on the current repair action. By performing multiple cycles of this model, we can estimate the life cycle cost of this asset that considers both repair decisions and further deterioration.

Different from the model in Figure 2, where an asset is assumed to be in a specific condition (hard evidence, for example, $100 \%$ in Good condition); the repair action cannot guarantee perfect maintenance. Hence, the repaired state is probabilistically distributed (soft evidence). The previous binary factorisation, therefore, becomes a special case when the repaired state is $100 \%$ in the Good condition. In fact, this only applies to the situation for complete replacement, while for imperfect maintenance with a probability distribution, we need to perform multiple levels of binary factorisations.

Assume asset can be rated among $\{n, \ldots, 1\}$ states, where state $n$ is the perfect state and State 1 is the worst state, we have $n-1$ transitions. By dividing the factorisation into $n-1$ blocks, where each block represents a factorisation process that start with one of the possible states. Given an intervention time, for state $i$, we perform a binary factorisation with the Markov property (for example, transit to state after $i-1$ only depends on transit to state after $i$ ) that evaluate whether the asset will deteriorate to state $\{i, \ldots, 1\}$. We model this process by a collection of temporal Boolean nodes from transit to state after i, to transit to State 1. In the block for state $i$, all temporal Boolean nodes are aggregated to form a temporal predicted state node representing the state distribution if the repaired state is in state $i$. The repaired state node contains a message about the probability distribution of each state. Together with the temporal predicted state nodes, they are aggregated to form an ultimate predicted state node representing the state distribution of this asset.

To define the CPT for the final predicted state node, we first need to determine the predicted probability for each state. For repaired state and the final predicted state, we have a collection of state distributions represented as $P_{\text {repair }, i}$ and $P_{\text {predict }, j}$ respectively, where $\sum_{i=1}^{n} P_{\text {repair }, i}=1$ and $\sum_{j=1}^{n} P_{\text {predict }, j}=1$. For the temporal predicted state that starts with repaired state $i$, its probability of deteriorate to state $j$ is 
represented as $p_{i, j}$, where $\sum_{j=1}^{i} p_{i, j}=1$, and $i \geq j$. Therefore, the probability of the ultimate predicted state in state $j$ is:

$$
P_{\text {predict }, j}=\sum_{i=j}^{n} p_{i, j} P_{\text {repair }, i}
$$

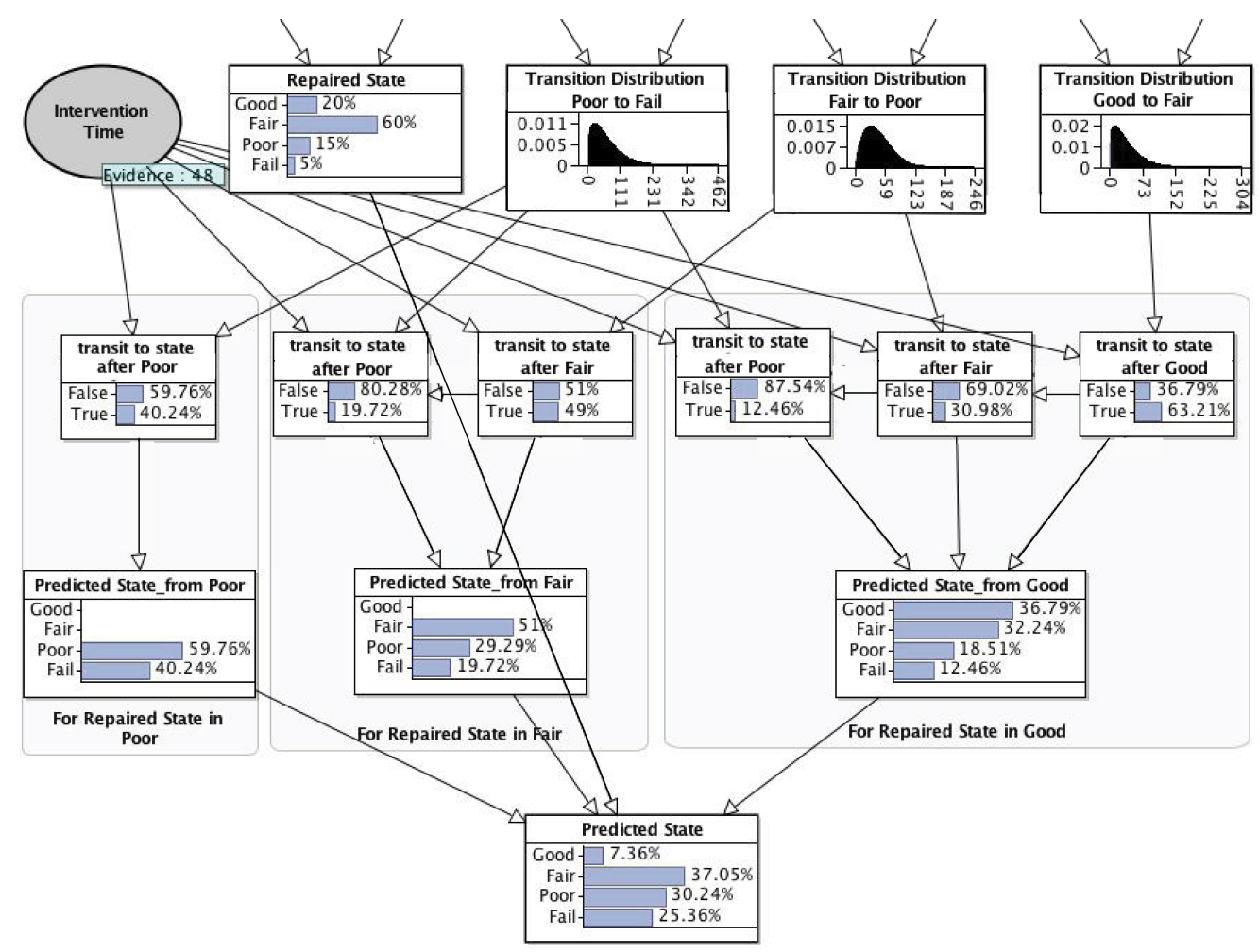

Figure 6: Multiple binary factorisations for the repaired state distributed probabilistically due to imperfect maintenance.

Figure 6 shows how we can decompose the factorisation for an asset that is rated by four states: Good, Fair, Poor and Fair. Hence, three transitions (the index $n-1=3$ ). For the repaired state that is in Good condition, followed the procedure in Section 3.1, its deterioration is binary factorised with three temporal nodes about its step-wise transitions given the observation of intervention time. These temporal nodes are gathered together to evaluate the further deterioration of asset under the circumstance that the repaired state is in Good condition. Similar 
procedures are performed to evaluate the deterioration of the asset state in Fair state and Poor state respectively. A collection of temporal predicted states for asset starts with various repaired states is linked, together with the probability distribution of the repaired state to infer the ultimate predicted state. For example, to evaluate the probability of this asset in Poor condition, Equation 3 is employed, where $j=2$ and $n=4$. We need to combine three situations, where each situation is multiplied by the probability of it being in that repaired state: in 48 months, the probability of repaired state in Good state deteriorates to Poor state, the probability of repaired state in Fair state deteriorates to Poor state, and the probability of repaired state in Poor state stays in the Poor state. Hence, we have $18.51 \% *$ $20 \%+29.29 \% * 60 \%+59.76 \% * 15 \%=30.24 \%$.

This model can be used to estimate the life cycle of asset maintenance and identify potential risk and expenditure. To further utilise this model, we can incorporate maintenance strategy to select optimal repair action and to decide intervention time interval. For example, by setting constraints to activate repair actions (e.g. some repair actions can be only used when the asset is in a particular state range), with objectives like minimum overall repair cost in the whole life cycle or ensuring asset state is above a certain level for safety reason, this forms a mathematical optimisation problem. It can be tackled by a range of techniques, such as heuristic algorithms, to decide which repair action to select at what time. Together with our model, we can perform analysis like optimal life cycle cost analysis to give more valuable suggestion when planning maintenance activities.

\subsection{Summary}

This section presents several $\mathrm{BN}$ models that can be used to manage asset maintenance-related decisions, including:

1. A model that predicts asset condition at a given time. This model incorporates binary factorisation to enable efficient inference with multiple sates. A second extension of the model is to use the condition of the components to infer the overall state of an asset.

2. Two repair decision Bayesian models. One can suggest repair action based on the historical actions, while the other one reasons the effect of selected repair action.

3. A model considers both asset deterioration and repair effectiveness over its life cycle. This model uses the repair model as an object so that, with 
multiple sequential objects, we can model the life cycle of an asset through several cycles of repair and further deterioration.

\section{Case Study Part 1: Inspection Decisions}

The challenge in making inspection decisions is to inspect a suitable asset at a suitable time. Understanding asset states from its deterioration is the foundation to recommend these decisions. Section 3.1 shows how we can model and inference multi-state asset with multiple components efficiently. This section provides applications of these models for a variety of inspection decisions using the NBI database from the US. Section 4.1 presents an application of condition prediction for a system with multiple components, and Section 4.2 shows how to reason inspection decisions from the predicted condition.

\subsection{Structural Deficiency Evaluation}

According to the Federal Highway Administration Bridge Preservation Guide, one of their common failure modes for bridge deficiency is structural deficient [32]. A deficient bridge has a higher possibility than a normal bridge in leading to a major structural event, such as bridge collapsion. This suggests the importance of imperative interventions. A non-culvert bridge is defined as SD either: i) a condition rating of 4 or less for any of its structures, including deck, superstructure and substructure, or ii) an appraisal rating of 2 or less for structural evaluation, or iii) an appraisal rating of 2 or less for waterway adequacy [25].

In this subsection, we like to use the predicted condition of each structure to estimate the probability of this bridge deteriorates into SD in a future time. The prediction can provide valuable information for decision making when planning inspection or repair. One of the criteria in resulting SD is any of its structures are considered as in Poor condition. Therefore, we can consider the structures of this bridge are arranged in series. Accordingly, we can model it using the OR gate from Section 3.1.2, where the system fails when any of the subsystems fall below condition 4.

The structural evaluation investigates if a bridge's loading capacity is significantly below its design standards. For structures other than culverts, the rating of structural evaluation is specified by the lowest rating between superstructure, substructure and a comparing rating between Average Daily Traffic (ADT) and inventory rating. ADT and inventory rating are both fixed variables, where ADT encodes the average daily traffic volume of the bridge, for example, 3222 vehicles; inventory rating represents the load level that allows a structure to operate for an 
indefinite period of time, for example, MS 12.5 (MS is a measure for loading capacity). By using the specification table provided in Weseman [25], we can gather the comparing rating, for example, a rating is coded as 6 given 3222 ADT and MS 12.5 inventory rating. Since the determination of the structural evaluation rating is the lowest score among those ratings, we can also model these criteria using an OR gate. A bridge is also SD when its waterway frequently overtops the bridge. The inspector often evaluates it from the on-site inspection. Similar to structures like a deck, we can learn its patterns from the inspection data and predicted the condition distribution of its adequacy in a future time.

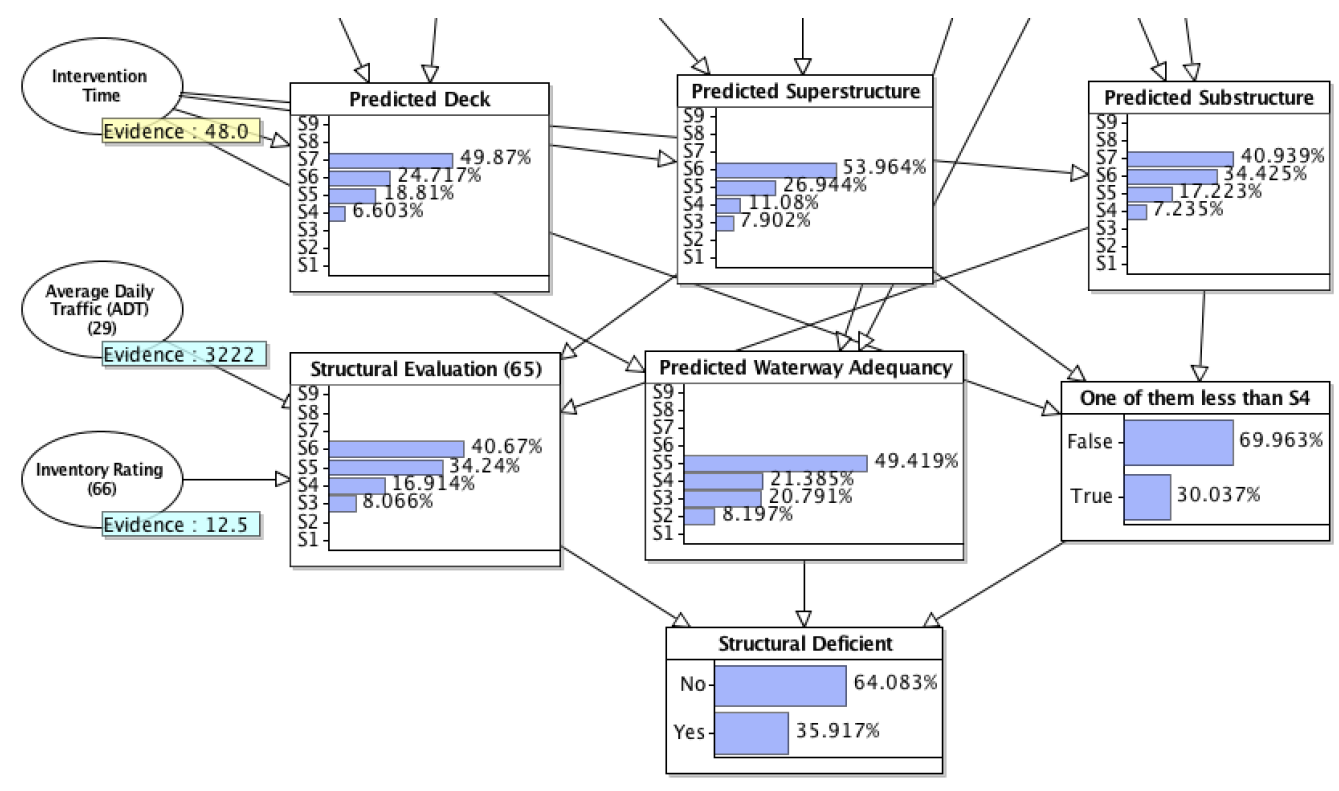

Figure 7: Structural deficiency evaluation.

Together, we can use a logical expression to combine the information using a Boolean node. It is true when one of the structures are rated 4 or less, or structural evaluation is rated 2 or less, or waterway adequacy is rated 2 or less. The example in Figure 7 is a non-culvert bridge that has an ADT of 3222, and a permitted loading capacity of MS 12.5. Its current conditions of deck, superstructure, substructure and waterway are rated as 7, 6, 7 and 5 respectively. This model predicts the probability of this bridge becomes structural deficient in the next 48 months is $35.92 \%$. This evaluation is useful in determining which bridge is in higher risk of becoming SD. Further, by reasoning this model backwardly, we can recommend inspection decisions, including the time to inspect, and the priority (among other 
bridges) for inspection, which will be discussed in the following subsection.

\subsection{Inspection Decisions Reasoning}

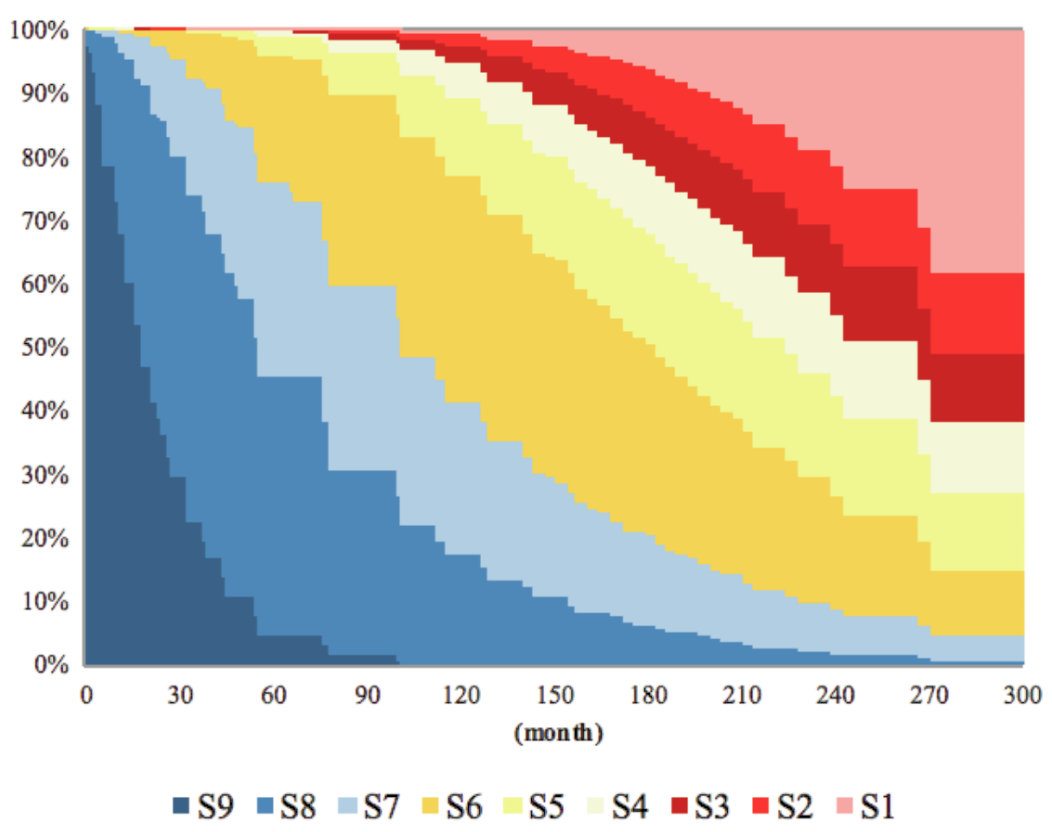

Figure 8: Deck condition distribution over time.

This subsection shows how we can make use of the condition prediction to support inspection decisions. Previously we've show that we can predict the condition distribution of a structure over a finite time frame. This structure could be a component, or an asset assembled by multiple components. Figure 8 shows an example of a new deck's predicted condition distribution over the next 300 months without interventions. The transition distributions are learned from the NBI dataset. With the increase in time, it is natural to see the trend of deterioration: the probability of S9 (perfect state) gradually decreases along with the increase of S8, and S8 decreases along with the increase of S7, and so on. For example, in around 90 months, the possibility of this deck still in S9 is nearly $0 \%$. However, sometimes, decision-makers are more interested in knowing how reliable this structure is over a period of time. We can use this information to identify assets with higher risk so we can plan accordant action to mitigate the risk.

For failure distributions like Weibull distribution are asymmetric. Reliability measures such as MTTF or MTBF use mean to describe failure mode are therefore 
not suitable. Instead, we would be more interested in the overall reliability. Failure rate function $f(t)$ measures how fast a structure will deteriorate. By taking its integral, we have its cumulative distribution function $F(t)$, which represents the failure probability that a random variable $t$ takes on a value less than or equal to $t$. Since we are more interested in how reliable the structure is, we can take the complementary cumulative distribution function to form the reliability function $R(t)$ as shown in Equation 4.

$$
R(t)=P(T \geq t)=\int_{t}^{\infty} f(x) d x=1-F(t)
$$

The reliability function measures the probability of an asset survives before moment $t$. To evaluate the reliability for a multi-state system, first, we need to define a level of acceptable conditions. For example, defined in FHWA [32], in the NBI dataset, structure states with a rating of 7,8 , or 9 are classified as Good condition, with a rating of 5 or 6 are Fair condition, and the rest are Poor condition (equivalent to SD). We can use this type of information to measure the reliability of an asset stays in these conditions without failing to other levels. A Boolean node (a constraint) is therefore added as a child node of the predicted condition. It has a logical expression stating that this node is true when the predicted condition is greater than the acceptable level (e.g. the predicted condition $\geq S 7$ for structure staying in Good condition). By observing this constraint as true and removing the observation on intervention time node, after inference, we get the reliability $R(t)$ of this structure from the posterior distribution of intervention time node.

Figure 9 shows four examples with two decks and each has two acceptable levels. One level has a higher standard that requires the structure in Good condition $(\geq S 7)$, and the other one accepts the structure in or above Fair condition $(\geq S 5)$. Naturally, within the same decks, the reliability of a deck in or above Fair condition is always higher than the one in Good condition. For example, in 48 months, Deck A condition $\geq S 5$ has a reliability 0.78 , condition $\geq S 7$ has a reliability of 0.61 ; Deck B condition $\geq S 5$ has a reliability 0.85 , condition $\geq S 7$ has a reliability of 0.63 . With the reliability, we can make inspection decisions including to prioritise assets for inspection and to determine a suitable time for inspection.

To prioritise assets for inspection based on their risks in deteriorating into an unacceptable level, we need to set a failure limit first. An example of a failure limit is set at 0.8 in Figure 9, where we want to ensure the reliability of the decks is above 0.8. To ensure deck condition $\geq S 7$, we can see both Deck A and B require intervention around 25 months. However, for condition $\geq S 5$, we can see 


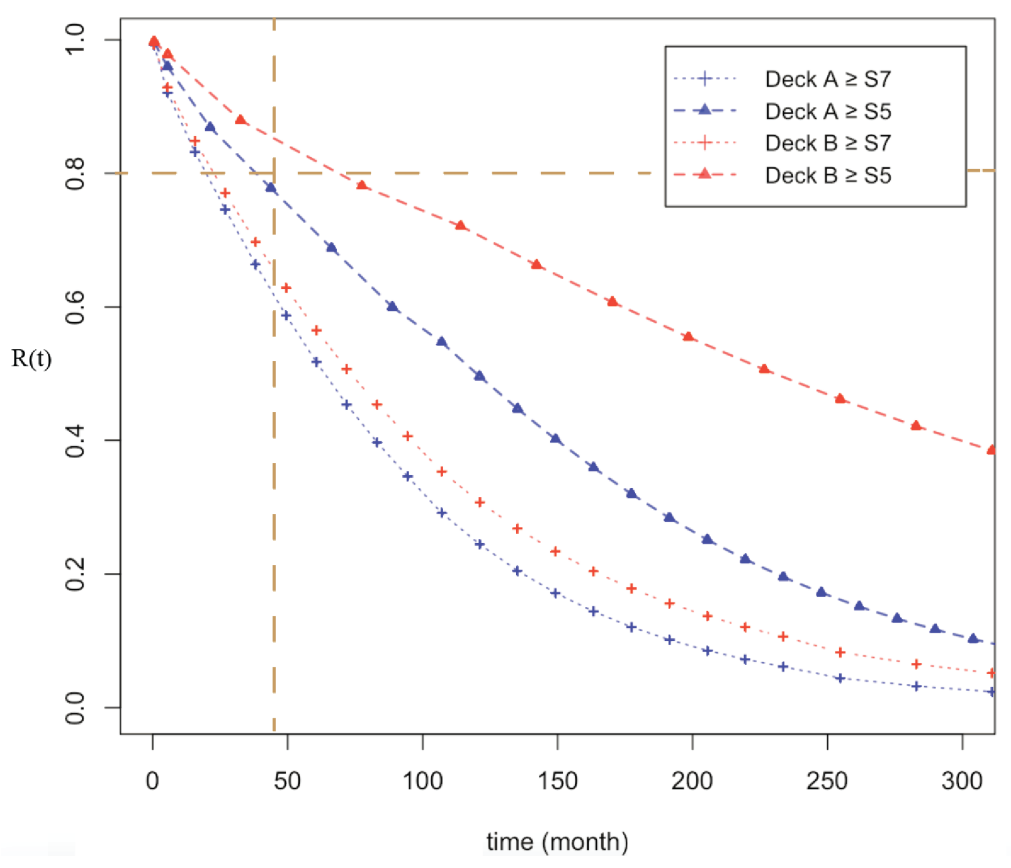

Figure 9: Reliability of decks.

an apparent distinction between Deck A and B: Deck A requires intervention at around 40 months while $B$ at around 70 months. This distinction reveals that Deck A has a higher risk in failing to Poor condition than Deck B. We can, therefore, to prioritise the inspection for Deck A over Deck B.

Another decision is to evaluate the suitability of inspection time. Most bridges in the US are inspected every 24 months, while in GB, bridges are visually inspected every 12 months and detailed inspected every 72 months. In current practice, most inspections are performed at fixed time intervals. We can improve this situation by tailoring inspection time for each structure using the reliability function inferred from the developed models. Using the same example in Figure 9 , to prevent deck condition from failing to Poor condition with a reliability of 0.8 , Deck A urges for an inspection to see if repair is needed at around 40 months, while Deck B is around 70 months.

\section{Case Study Part 2: Repair Decisions}

Given an asset condition, we may want to recommend repair action to satisfy a range of objectives. It is a challenge since a repair action may only be suitable 
for a specific condition of a specific asset. Different repair actions may also come with different restoring capability and cost. With the observational model from Section 3.2.1, by extending it with a set of constraint nodes, we can reason the frequency of each repair decision made in history. The decision maker can refer it as what action is usually taken in history. Another decision is to evaluate the effectiveness of maintenance, which is enabled by the intervention model from Section 3.2.2. This can help decision maker understand the potential repaired condition and resource usage.

These maintenance decisions are introduced aligned with the background of bridge deck maintenance in the US. Table A.1 (in Appendix) from Michigan Department of Transportation [33] is a typical guideline for maintenance of concrete bridge deck that has epoxy coated rebar. This table is built upon deck deterioration data and expert knowledge from experienced engineers. Together with the deck condition, this table can provide guidance for repair action selection.

The bridge deck condition is evaluated by the condition of its top surface and bottom surface. Available repair actions vary from its current condition. For example, deck patching is only applicable when its top surface is rated not less than State 5 and bottom surface not less than State 4 . The repairing capacity also varies, for example, deck patching can improve the condition of a deck top surface by 1 point with an expected service life of 3 to 10 years. While for shallow concrete overlay, it can improve top surface to State 9 or 8 with an expected service life of 20 to 25 years when it was rated at a State 4 or 5; 10 years when it was rated at a state less than 3 . However, most actions cannot repair deck bottom surface it can only be replaced. Additionally, Table A.2 (in Appendix) shows the related repaired costs listed in Winn and Burgueo [34], which were gathered from the author's personal communication with the engineers. Information about the area of an individual deck can be retrieved from the NBI database: structure length is recorded in Item 49 and deck width is in Item 52 (see Weseman [25] for details).

\subsection{Repair Action Recommendation}

The repair option depends on the condition of both deck top surface and bottom surface. Its CPT is derived from Table A.1, where each repair action has an uniform distribution if more than one option is applicable under the required conditions. For example, if the condition of deck top surface is rated at State 5 and the bottom surface is rated at State 4 from the most recent inspection, the applicable actions include deck patching, shallow concrete overlay, and HMA overlay with a waterproofing membrane. These three actions each has a $1 / 3$ probability, and other actions have 0 in the CPT. The CPT for the repaired condition is generated 


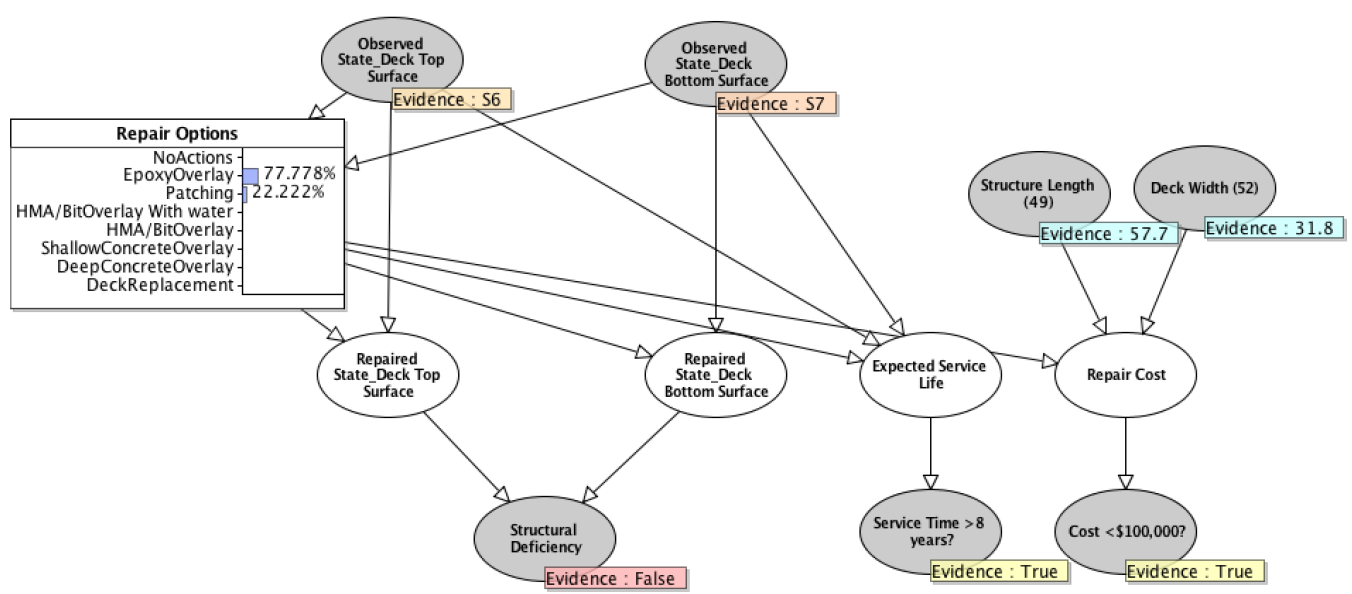

Figure 10: Maintenance action recommendation.

by columns under potential result in the table, with an uniform distribution if there is more than one possible result. For example, if an epoxy overlay is performed, the top surface will restore to condition 9 with a 0.5 probability and condition 8 with a 0.5 probability. However, the bottom surface will stay as the same condition from the last inspection since this repair action has no effect on it. Similarly, the expected service life is modelled using a partitioned expression from the repair actions and top and bottom deck surface conditions. For example, shallow concrete overlay action on deck top surface with a condition 4 and bottom surface with a condition 4 , it has an anticipated service life that expressed by a uniform distribution with a lower bound of 20 and upper bound of 25 . While for a deck with a condition 3 on its top surface, its anticipated life is only 10 years.

To reason repair action that was usually taken, three types of constraints are modelled, showed in Figure 10. These constraints are all modelled using Boolean nodes with logical expressions. The structural deficiency is true when either the repaired deck top or bottom surface has a rating less or equal to 4 . The decision makers set the expected service life about how many years they anticipate the deck will survive. The cost of repair action can be modelled using a partitioned expression, where each repair option is modelled by the compound of cost per unit (see Table A.2), and its area. Decision makers suggest the cost constraint about how much budget is allowed.

The example in Figure 10 is based on a deck with its top surface that is inspected with a rating of 6 and the bottom surface with a rating of 7 . The length of this deck is $57.7 f t$ and width is $31.8 f t$. The decision maker does not want 
the repaired deck becomes structurally deficient and wishes the service time to be greater than eight years with a cost less than $\$ 100,000$. After inference of the model, in history, under the same constraints, $77.78 \%$ of maintainers repaired with epoxy overlay and $22.22 \%$ repaired with deck patching. This model rules out repair options that weren't taken by other maintainers in the past automatically, which makes the decision-making process for evaluating repair action easier. But if we want to estimate the effectiveness of different maintenance action only, we need an intervention model.

\subsection{Repair Action Evaluation}

Intervention analysis can help decision maker prioritise repair actions depends on the evidence. In Table A.1, interventions for deck condition contain both imperfect and perfect maintenance. For example, HMA Cap action can imperfectly restore deck top surface to State 8 with a 0.5 probability and State 9 with a 0.5 probability while the replacement is a perfect repair action that restore both deck top and bottom surface to a new condition. To reason the effectiveness of repair actions directly, in the intervention model, we need to manipulate the intervention independently from its causes.

To convert an observational model into an intervention model, we only need to remove the links from causes (the two observed state variables about deck surface in Figure 10) that enter the intervention variable. The reason is, in the observational model, the use of repair action explains the historical frequency of actions taken under the observed conditions [30]. The repair options become a set of maintenance suggestions that are distributed probabilistically inferred from the posterior probabilities. While in the intervention phase, the repair action should be selected: only one action can be performed in each intervention. Only by removing the links from the parents we can remove the implication from past decisions, and directly show the effectiveness of repair action on manipulating structure repaired condition. At the same time, the constraints from the observational model all become unobservable in the intervention model to show the associated result of repair actions.

\section{Case Study Part 3: Maintenance Planning}

Planning maintenance over a finite time horizon is valuable in the maintenance strategy design phase. It can benefit the allocation of resource and investment decision-makings. This section shows how to utilise the developed models to 
evaluate strategy effectiveness. We illustrate this with a simplified maintenance strategy for life-cycle cost analysis.

Since inspection and repair activities for bridges are performed periodically with a time interval, to perform life cycle cost analysis over a finite time horizon on bridges, we can discretise the time horizon into several cycles. For simplicity, we illustrate this idea using a fixed interval of every two years over a hundredyear lifetime. However, notes that it is possible to implement with a dynamic intervention schedule by integrating with an optimisation technique.

In each intervention cycle, we model the repair decision-making process together with its deterioration process. Intervention model from Section 5.2 is employed to infer the effectiveness of repair. To model multiple intervention cycles sequentially, we can use the predicted structure condition in the current intervention as the input for the next intervention cycle. This can be modelled by multiple sequentially arranged $\mathrm{BN}$ objects developed from Section 3.3, where the predicted state at intervention $i$ will become the prior of previous state node at intervention $i+1$, and used to reason the repaired condition as well as to predict its further deterioration as shown in Figure 11. In this model, the condition of a structure is a future condition that can cannot be directly observed but predicted with a probabilistically distributed condition using the deterioration learning models. The employment of multiple binary factorisations discussed in Section 3.3 resolves the problem raised from the inference complexity. The cost of all actions is recorded using a cumulative cost function and sequentially becomes the prior of the previous repair cost node in the next intervention cycle.

Within an asset's life cycle, a structure is usually maintained multiple times. The choice of repair option is a decision node that relies on the anticipated conditions post-repair from the last intervention cycle. A maintenance strategy is used to decide what action we should take at what time. To convey the idea of how to use the proposed method to evaluate the effectiveness of maintenance strategy, we implemented several simplified strategies. Each strategy only consists of three repair options: if the deck conditions fall into the trigger condition of the repair options, one type of repair action from Table A.1 will be performed; otherwise, if the deck conditions satisfy the trigger condition of replacement, deck replacement will be performed; otherwise, no action will be taken. 


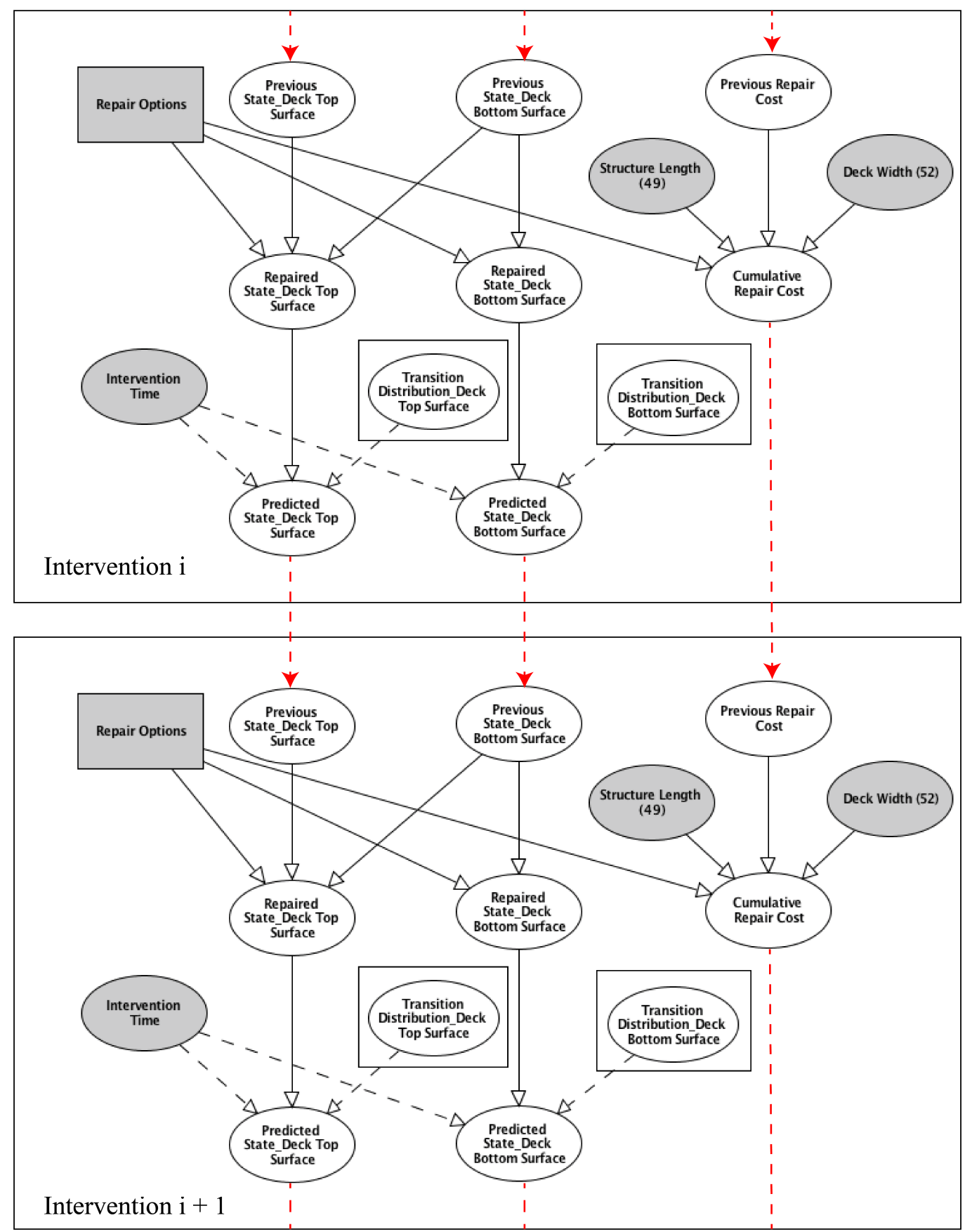

Figure 11: Life-cycle cost analysis from multiple sequentially organised BN objects. 


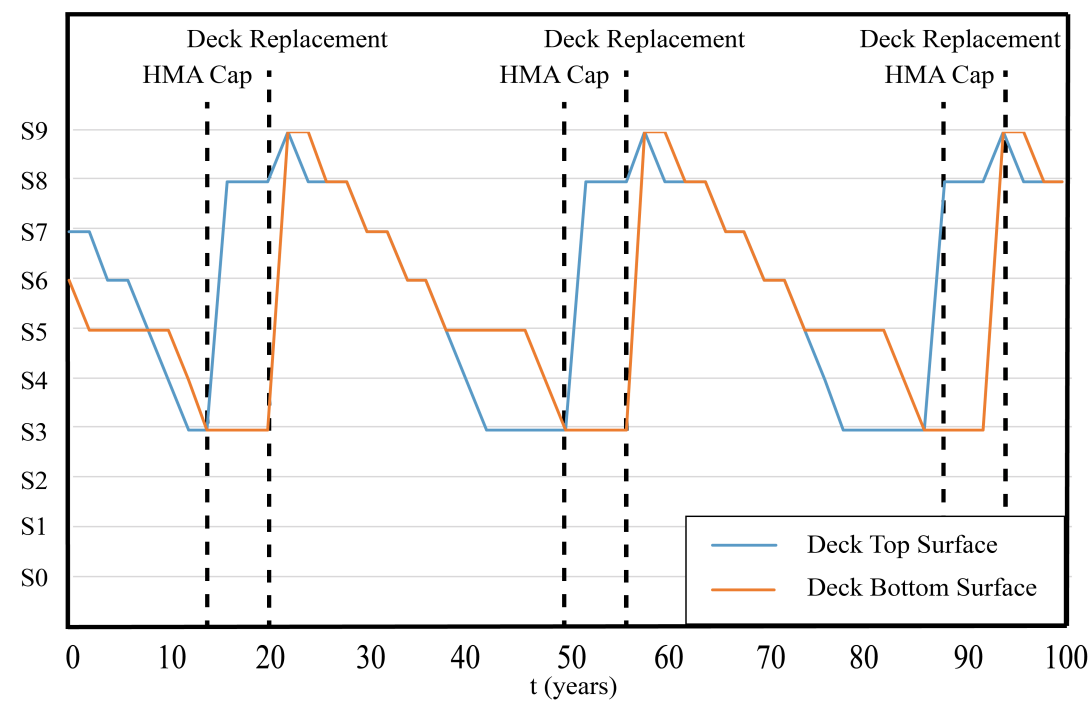

Figure 12: Deck top and bottom surface conditions with repairs in a 100 years horizon.

Figure 12 shows the change of deck top and bottom surface conditions (with an initial state of S7 and S6 respectively) over the next 100 years. The maintenance strategy implemented here comprises of maintenance action HMA Cap and deck replacement. If the state of deck top surface deteriorates to a state of 4 or 5 and the bottom surface is in a state of 2 or 3 , or the deck top surface deteriorates to a state less or equal to 3 , and the bottom surface is in a state of 2 or 3, HMA Cap is triggered. Since HMA Cap is a temporal repair action with a short expected service life of 1 to 3 years, as the footnote in Table A.1 instructed, the deck is scheduled for replacement in the next five years. For example, in year 14, the top surface is predicted with a state of 3 same as the bottom surface. Therefore, an HMA Cap is performed that restores the condition of the top surface to 8 while the bottom surface remains the same. After five years, the deck is replaced with both top and bottom surface back to S9. In the next 100 years, implementing this maintenance strategy requires three HMA Cap actions and three replacements. Together with the cost presented in Table A.2, for a $57.7 \mathrm{ft}$ length and $31.8 \mathrm{ft}$ width deck, we estimated it has an overall repair cost of $\$ 392,201.33$. 


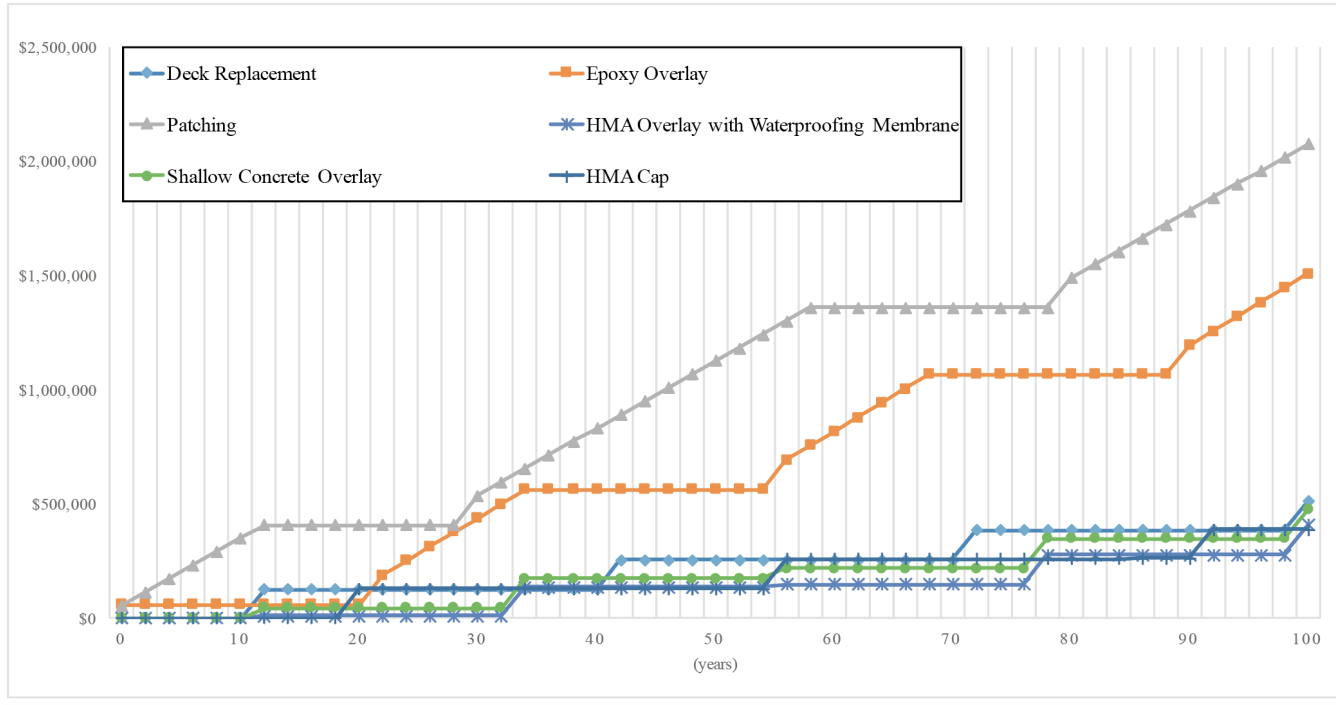

Figure 13: Repair cost with different maintenance strategy in the next 100 years.

Different repair action based strategies are evaluated, and their cumulative costs are presented in Figure 13. Epoxy overlay and patching are the two most costly strategies since they prefer to repair deck top surface while they are still in fair conditions. Other approaches share similar costs. Among them, HMA Overlay with waterproofing membrane strategy has the lowest cost. Though deck replacement strategy (only repair with replacement) has a lower cost than some strategies, the deck stays in poor condition most of the time. In some cases, keeping structures staying in better conditions throughout its lifetime, even in the cost of a higher expenditure, is still desired due to safety and reliability reason.

The repair decisions in this example are triggered by the structures' conditions only. However, in practice, usually there are more constraints when deciding the maintenance decisions, such as repair action availability, cost and reliability. This form a multi-criteria decision problem. Our model gives a framework to integrate deterioration prediction with repair decisions. Together with an optimisation technique, by giving a set of constraints, we can make optimal decisions for maintenance. For example, together with the model shown in Figure 11, we can use a Genetic Algorithm to generate a maintenance strategy that takes both asset deterioration and maintenance effectiveness into consideration. We can therefore optimally select different maintenance actions to restore an asset condition to a suitable condition at a suitable time with an objective of minimum cost and high level of reliability. The strategy can be implemented in the decision node repair 
options in Figure 11 and to estimate its life cycle cost for maintenance planning.

\section{Conclusion}

This paper presents a series of novel BN models and shows the uses of them in supporting a range of maintenance-related decisions. These models are further applied using the case studies of bridge maintenance in the US. These models support decisions in several cases including:

1. Structural evaluation of an asset that is assembled by multiple components, where the condition of each component is predicted from multiple deterioration models. With the posterior distribution from the prediction models, we can perform reliability analysis to prioritise asset to inspect and to suggest inspection time given a reliability threshold.

2. Given an inspection result, we can infer what repair action is usually performed in history, or we can show the effectiveness of different maintenance actions. We can use them as a reference when making repair decisions.

3. By combining models of repair and deterioration over several maintenance cycles, we can evaluate the effectiveness of different maintenance strategy over a time horizon. We can use it to perform life cycle analysis.

We also point out the potential to combine the developed models with an optimisation technique when planning maintenance activities. Multi-criteria and multi-objective decision making have been intensively studied in maintenance problems with satisfying performance (see de Almeida et al. [35] for a review). As a future step, with constraints like cost, asset state and reliability, and objectives like a certain level of reliability or minimum cost, we would like to find the optimal selection of repair over some time.

\section{Acknowledgements}

This is supported by EPSRC (EP/P009964/1: PAMBAYESIAN). We also thank Agena for use of their software AgenaRisk. 


\section{References}

[1] Organisation for Economic Co-operation and Development (OECD), Infrastructure to 2030: Telecom, land transport, water and electricity, OECD Publishing, 2006.

[2] A. Heng, S. Zhang, A. C. Tan, J. Mathew, Rotating machinery prognostics: State of the art, challenges and opportunities, Mechanical Systems and Signal Processing 23 (2009) 724-739.

[3] D. M. Frangopol, K.-Y. Lin, A. C. Estes, Life-cycle cost design of deteriorating structures, Journal of Structural Engineering 123 (1997) 1390-1401.

[4] A. Lisnianski, I. Frenkel, A. Karagrigoriou, Recent Advances in Multi-state Systems Reliability: Theory and Applications, Springer, 2017.

[5] N. Khakzad, F. Khan, P. Amyotte, Safety analysis in process facilities: Comparison of fault tree and Bayesian network approaches, Reliability Engineering \& System Safety 96 (2011) 925-932.

[6] Q. Mahboob, A Bayesian Network methodology for railway risk, safety and decision support, Ph.D. thesis, Technische Universitt Dresden, 2013.

[7] M. A. Cesare, C. Santamarina, C. Turkstra, E. H. Vanmarcke, Modeling Bridge Deterioration with Markov Chains, Journal of Transportation Engineering 118 (1992) 820-833.

[8] Y. Jiang, M. Saito, K. C. Sinha, Bridge Performance Prediction Model Using the Markov Chain, Transportation Research Record 1180 (1988) 25-32.

[9] Y. Shafahi, R. Hakhamaneshi, Application of a Maintenance Management Model for Iranian Railways Based on the Markov Chain and Probabilistic Dynamic Programming, International Journal of Science and Technology. Transaction A: Civil Engineering 16 (2009) 87-97.

[10] E. L. Droguett, M. das Chagas Moura, C. M. Jacinto, M. F. Silva Jr, A semi-markov model with bayesian belief network based human error probability for availability assessment of downhole optical monitoring system, Simulation Modelling Practice and Theory 16 (2008) 1713-1727.

[11] P. C. Yianni, D. Rama, L. C. Neves, J. D. Andrews, D. Castlo, A Petri-netbased modelling approach to railway bridge asset management, Structure and Infrastructure Engineering 13 (2017) 287-297.

[12] T. Murata, Petri nets: Properties, analysis and applications, Proceedings of the IEEE 77 (1989) 541-580.

[13] P. Weber, G. Medina-Oliva, C. Simon, B. Iung, Overview on Bayesian networks applications for dependability, risk analysis and maintenance areas, Engineering Applications of Artificial Intelligence 25 (2012) 671-682. 
[14] J. Andrews, A modelling approach to railway track asset management, Proceedings of the Institution of Mechanical Engineers, Part F: Journal of Rail and Rapid Transit 227 (2013) 56-73.

[15] B. Le, Modelling railway bridge asset management, $\mathrm{Ph} . \mathrm{D}$. thesis, University of Nottingham., 2014.

[16] B. Le, J. Andrews, Petri net modelling of bridge asset management using maintenance-related state conditions, Structure and Infrastructure Engineering 12 (2016) 730-751.

[17] G. Chalkidis, M. Nagasaki, S. Miyano, High performance hybrid functional petri net simulations of biological pathway models on cuda, IEEE/ACM Transactions on Computational Biology and Bioinformatics 8 (2010) 15451556.

[18] P. C. Yianni, L. C. Neves, D. Rama, J. D. Andrews, Accelerating petrinet simulations using nvidia graphics processing units, European Journal of Operational Research 265 (2018) 361-371.

[19] M. I. Rafiq, M. K. Chryssanthopoulos, S. Sathananthan, Bridge condition modelling and prediction using dynamic Bayesian belief networks, Structure and Infrastructure Engineering 11 (2015) 38-50.

[20] B. Littlewood, J. L. Verrall, A bayesian reliability model with a stochastically monotone failure rate, IEEE Transactions on Reliability 23 (1974) 108-114.

[21] D. Marquez, M. Neil, N. Fenton, A new Bayesian network approach to reliability modelling, in: Mathematical Methods in Reliability (MMR07).

[22] Agena Ltd, AgenaRisk: Bayesian network software for risk analysis and decision making, 2018. https://www.agenarisk.com, last accessed 11 October 2020.

[23] H. Zhang, D. W. R. Marsh, Generic bayesian network models for making maintenance decisions from available data and expert knowledge, Proceedings of the Institution of Mechanical Engineers, Part O: Journal of Risk and Reliability 232 (2018) 505-523.

[24] H. Zhang, D. W. R. Marsh, Multi-state deterioration prediction for infrastructure asset: Learning from uncertain data, knowledge and similar groups, Information Sciences 529 (2020) 197-213.

[25] W. Weseman, Recording and coding guide for the structure inventory and appraisal of the nation's bridges, 1995. Report to United States Department of Transportation, Federal Highway Administration, USA.

[26] D. Marquez, M. Neil, N. Fenton, Improved reliability modeling using Bayesian networks and dynamic discretization, Reliability Engineering \& 
System Safety 95 (2010) 412-425.

[27] M. Neil, X. Chen, N. Fenton, Optimizing the calculation of conditional probability tables in hybrid Bayesian networks using binary factorization, IEEE Transactions on Knowledge and Data Engineering 24 (2012) 13061312.

[28] B. Meder, Y. Hagmayer, M. R. Waldmann, Understanding the causal logic of confounds, in: Twenty-Eighth Annual Conference of the Cognitive Science Society, pp. 579-584.

[29] R. Silva, Observational-interventional priors for dose-response learning, in: Advances in Neural Information Processing Systems, pp. 1561-1569.

[30] A. C. Constantinou, B. Yet, N. Fenton, M. Neil, W. Marsh, Value of information analysis for interventional and counterfactual Bayesian networks in forensic medical sciences, Artificial Intelligence in Medicine 66 (2016) 41-52.

[31] N. Fenton, M. Neil, Risk assessment and decision analysis with Bayesian networks, CRC Press, 2018.

[32] FHWA, Bridge preservation guide - maintaining a resilient infrastructure to preserve mobility, U.S. Department of Transportation: Federal Highway Administration (2018).

[33] Michigan Department of Transportation, Bridge deck preservation matrix, 2008. Report to Michigan Department of Transportation.

[34] E. K. Winn, R. Burgueo, Development and validation of deterioration models for concrete bridge decks. Phase 1: Artificial intelligence models and bridge management system, 2013. Research Report to Michigan DOT, Report No. CEE-RR 2013/01.

[35] A. T. de Almeida, R. J. P. Ferreira, C. A. V. Cavalcante, A review of the use of multicriteria and multi-objective models in maintenance and reliability, IMA Journal of Management Mathematics 26 (2015) 249-271. 


\section{Appendix A}

Table A.1: Bridge Deck Preservation Matrix - Decks with epoxy coated rebar [33].

\begin{tabular}{|c|c|c|c|c|c|}
\hline \multicolumn{2}{|c|}{ Deck Condition } & \multirow[t]{2}{*}{ Repair Options } & \multicolumn{2}{|c|}{ Potential Result } & \multirow{2}{*}{$\begin{array}{l}\text { Expected Life } \\
\text { (years) }\end{array}$} \\
\hline Top & Bottom & & Top & Bottom & \\
\hline \multirow[t]{3}{*}{$\geq 5$} & $\mathrm{~N} / \mathrm{A}$ & Hold or Seal Cracks & No Change & No Change & 1 to 4 \\
\hline & $>5$ & Epoxy Overlay & 8,9 & No Change & 10 to 15 \\
\hline & $\geq 4$ & Deck Patch & Up by 1 & No Change & 3 to 10 \\
\hline \multirow[t]{3}{*}{4 or 5} & 4 & Shallow Concrete Overlay & 8,9 & No Change & 20 to 25 \\
\hline & & $\begin{array}{l}\text { HMA Overlay with Water- } \\
\text { proofing Membrane }\end{array}$ & 8,9 & No Change & 8 to 10 \\
\hline & 2 or 3 & HMA Cap $^{\mathrm{a}}$ & 8,9 & No Change & 2 to 4 \\
\hline \multirow[t]{4}{*}{$\leq 3$} & 4 or 5 & Shallow Concrete Overlay & 8,9 & No Change & 10 \\
\hline & & $\begin{array}{l}\text { HMA Overlay with Water- } \\
\text { proofing Membrane }\end{array}$ & 8,9 & No Change & 5 to 7 \\
\hline & 2 or 3 & HMA Cap ${ }^{\mathrm{a}}$ & 8,9 & No Change & 1 to 3 \\
\hline & & $\begin{array}{l}\text { Replacement with Epoxy } \\
\text { Coated Rebar Deck }\end{array}$ & 9 & 9 & $60+$ \\
\hline
\end{tabular}

${ }^{\text {a }}$ Hot Mix Asphalt (HMA) Cap for deck improvement. After HMA Cap, deck replacement should be planned in the next 5 years.

Table A.2: Repair cost for bridge deck with epoxy coated rebar [34].

\begin{tabular}{lll}
\hline Repair Options & Unit Price & Notes \\
\hline \hline Patching & $\$ 32 / f t^{2}$ & Includes hand chipping \\
Epoxy Overlay & $\$ 3.80 / f t^{2}$ & \\
HMA Overlay/Cap & $\$ 1.25 / f t^{2}$ & $\begin{array}{l}\text { An extra } \$ 5 / \mathrm{ft}^{2} \text { if the repair includes waterproofing } \\
\text { membrane }\end{array}$ \\
$\begin{array}{lll}\text { Shallow Concrete Overlay } \\
\text { Deck Replacement }\end{array}$ & $\$ 25 / f t^{2}$ & $\begin{array}{l}\text { Includes joint replacement and hydro } \\
\text { Includes removal of the old deck and new railings }\end{array}$ \\
\hline
\end{tabular}

